Check for updates

Cite this: Phys. Chem. Chem. Phys., 2021, 23, 4882

Received 21st December 2020, Accepted 11th February 2021

DOI: 10.1039/d0cp06587k

rsc.li/pccp

\section{The effect of ionic defect interactions on the hydration of yttrium-doped barium zirconate $\uparrow$}

\begin{abstract}
Sebastian Eisele, (D) ${ }^{\text {ab }}$ Fabian M. Draber (D) ${ }^{b}$ and Steffen Grieshammer (D) *ab
Hydrated acceptor-doped barium zirconate is a well-investigated proton conductor. In the analysis of most experimental studies, an ideal defect model is applied to fit the measured hydration data and obtain corresponding enthalpies and entropies. However, the data show a distinct deviation from ideal behaviour and thus defect interactions cannot be neglected. In the present contribution, the thermodynamics of water uptake into the yttrium-doped bulk material are investigated on the microscopic level with regards to ionic defect interactions. Metropolis Monte Carlo simulations using interaction models from first-principles energy calculations are applied to obtain an estimation of the free energy of interaction. The present results indicate that the ionic defect interactions are the primary reason for the non-ideality observed in experiments with varying yttrium fraction, proton fraction, and temperature. The activity coefficient quotients for the mass action law are obtained, which connect the ideal and real model and are of relevance to data evaluation and theoretical calculations.
\end{abstract}

\section{Introduction}

Barium-zirconate $\mathrm{BaZrO}_{3}$ (BZO) is a well-investigated highperformance proton conductor with potential application as an electrolyte in protonic ceramic fuel cells (PCFC). The bulk material crystalizes in the $\mathrm{ABO}_{3}$ perovskite structure with space group $P m \overline{3} m$ (No. 221). To obtain an ion conductor, BZO is doped with an acceptor on the $\mathrm{Zr}$ site, here trivalent yttrium, resulting in mobile oxygen vacancies $\mathrm{V}_{\mathrm{O}}^{\bullet \bullet}$ according to reaction (1) given in Kröger-Vink notation. ${ }^{1}$

$$
\mathrm{Y}_{2} \mathrm{O}_{3}+2 \mathrm{BaO} \stackrel{\mathrm{BaZrO}_{3}}{\longrightarrow} 2 \mathrm{Ba}_{\mathrm{Ba}}^{\times}+2 \mathrm{Y}_{\mathrm{Zr}}^{\prime}+5 \mathrm{O}_{\mathrm{O}}^{\times}+\mathrm{V}_{\mathrm{O}}^{\bullet \bullet}
$$

Hydration of the doped material $\mathrm{BaZr}_{1-x} \mathrm{Y}_{x} \mathrm{O}_{3-0.5 x}$ (BZY) partially refills the oxygen vacancies. This yields mobile interstitial protons $\mathrm{H}_{\mathrm{i}}^{\bullet}$ which are bound to the oxygen ions forming $\mathrm{OH}_{\mathrm{O}}^{\bullet}$ defects according to the equilibrium reaction (2).

$$
\mathrm{V}_{\mathrm{O}}^{\bullet \bullet}+\mathrm{O}_{\mathrm{O}}^{\times}+\mathrm{H}_{2} \mathrm{O}(\mathrm{g}) \rightleftharpoons 2 \mathrm{OH}_{\mathrm{O}}^{\bullet}
$$

Under the assumption of an ideal solution, the mass action law for reaction (2) can be written as eqn (3). The corresponding equilibrium constant $K_{0}(T)$ can then be calculated by the

${ }^{a}$ Helmholtz-Institut Münster (IEK-12), Forschungszentrum Jülich GmbH, Corrensstr. 46, 48149 Münster, Germany. E-mail: s.grieshammer@fz-juelich.de

${ }^{b}$ Institute of Physical Chemistry, RWTH Aachen University, Landoltweg 2, 52056 Aachen, Germany

$\dagger$ Electronic supplementary information (ESI) available. See DOI: 10.1039/ d0cp06587k standard enthalpy $\Delta h^{\circ}$ and standard entropy $\Delta s^{\circ}$ of hydration according to eqn (4) for non-interacting defects. Here, $p^{\circ}$ is a standard pressure of 1 bar.

$$
\begin{gathered}
K_{0}(T)=\frac{p^{\circ}}{p_{\mathrm{H}_{2} \mathrm{O}}} \cdot \frac{\left[\mathrm{OH}_{\mathrm{O}}^{\bullet}\right]^{2}}{\left[\mathrm{~V}_{\mathrm{O}}^{\bullet \bullet}\right]\left[\mathrm{O}_{\mathrm{O}}^{\times}\right]} \\
K_{0}(T)=\exp \left(\frac{-\Delta h^{\circ}}{k_{\mathrm{B}} T}\right) \exp \left(\frac{\Delta s^{\circ}}{k_{\mathrm{B}}}\right)
\end{gathered}
$$

In recent years, multiple studies obtaining $\Delta h^{\circ}$ and $\Delta s^{\circ}$ by fitting eqn (4) to experimental data accumulated strong evidence that the assumption of an ideal solution is mostly inaccurate. ${ }^{2-6}$ It was repeatedly shown that data obtained from experiments investigating the proton content, such as thermogravimetry, are not well described by eqn (3) and (4) when the bulk material is subjected to larger variations of temperature or water partial pressure. Kreuer obtained satisfactory fitting results for the hydration isobars only by considering an effective dopant concentration indicating that the ideal solution model is too simple. ${ }^{7}$

The strong dependency on proton concentration indicates a critical influence of the ionic defect interactions on the hydration equilibrium in BZY. Since the proton diffusivity in BZY is affected by both yttrium fraction and proton content, the hydration levels under specific conditions are of primary interest from a practical point of view. Several theoretical studies calculated the hydration enthalpy of $\mathrm{BZY} ;{ }^{8,9}$ however, the dependence on defect concentration is not directly investigated in these studies. 
One prominent effect is proton trapping which describes the tendency of $\mathrm{H}_{\mathrm{i}}^{\bullet}$ defects to accumulate at the $\mathrm{Y}_{\mathrm{Zr}}^{\prime}$ defects due to the attractive $\mathrm{Y}_{\mathrm{Zr}}^{\prime}-\mathrm{H}_{\mathrm{i}}^{\bullet}$ interactions at short range which affects the proton diffusivity in $\mathrm{BZY} .{ }^{10,11}$ This proton-trapping effect should also affect the hydration behaviour and is expected to be especially important at low temperatures. ${ }^{12,13}$ Analogous trapping of $\mathrm{V}_{\mathrm{O}}^{\bullet \bullet}$ at the $\mathrm{Y}_{\mathrm{Zr}}^{\prime}$ defects is found as a result of strongly attractive $\mathrm{Y}_{\mathrm{Zr}}^{\prime}-\mathrm{V}_{\mathrm{O}}^{\bullet \bullet}$ interactions at short range.

On the other hand, $\mathrm{V}_{\mathrm{O}}^{\bullet \bullet}-\mathrm{H}_{\mathrm{i}}^{\bullet}$ and $\mathrm{H}_{\mathrm{i}}^{\bullet}-\mathrm{H}_{\mathrm{i}}^{\bullet}$ pairs show strong repulsion at short range. The proton interstitials exist as $\mathrm{OH}_{\mathrm{O}}^{\bullet}$ defects $^{3,14-16}$ with a covalent $\mathrm{O}-\mathrm{H}$ bond. This implies that no proton is directly located at a vacant oxygen site and that no water-like molecules with more than one proton at an oxygen exist. ${ }^{11,16}$ The two remaining repulsive defect interactions $\mathrm{V}_{\mathrm{O}}^{\bullet \bullet}-\mathrm{V}_{\mathrm{O}}^{\bullet \bullet}$ and $\mathrm{Y}_{\mathrm{Zr}}^{\prime}-\mathrm{Y}_{\mathrm{Zr}}^{\prime}$ are expected to have two effects: (i) the $\mathrm{Y}_{\mathrm{Zr}}^{\prime}$ defects may be partially ordered if low sintering temperatures are used; and (ii) accumulating a large number of $\mathrm{V}_{\mathrm{O}}^{\bullet \bullet}$ defects at a single $\mathrm{Y}_{\mathrm{Zr}}^{\prime}$ defect is unfavourable.

Overall, the magnitude of these effects depends on the $\mathrm{OH}_{\mathrm{O}}^{\bullet}$ concentration and thus the hydration of BZY must be expected to be a non-ideal process. The defect interactions can have a profound influence on the enthalpic and entropic terms of eqn (3) and consequently the equilibrium state of BZY. As the balance between attractive and repulsive interactions depends directly on the $\mathrm{OH}_{\mathrm{O}}^{\bullet}$ concentration, it can be expected that there are two concentration regimes where the enthalpic term favors or hinders hydration, respectively. In addition, defect interactions are expected to cause a significant decrease in the configurational entropy by increasing the ordering of the defects in the system with decreasing temperature. Here, both attractive and repulsive interactions cause ordering so that an a priori prediction about the dependency on the $\mathrm{OH}_{\mathrm{O}}^{\bullet}$ concentration is difficult.

In this article, we apply a combination of density functional theory (DFT) first-principles energy calculations and large-scale Metropolis Monte Carlo ${ }^{17,18}$ (MMC) simulations to model the hydration of BZY with interacting defects and estimate the influence of the ionic defect interactions on the hydration equilibrium in the bulk material. We obtain the interaction contribution to the free energy of the system $F_{\text {int }}$ as a function of temperature $T$, yttrium doping $x_{\mathrm{Y}}$, and proton molar fraction $\left[\mathrm{OH}_{\mathrm{O}}^{\bullet}\right]$, through multistage MMC sampling as introduced by Valleau and Card. ${ }^{19}$ This approach was successfully applied to the reduction of ceria ${ }^{20}$ and here we aim to analyse the experimental findings for hydration equilibrium states of the BZY bulk material with interacting ionic defects through analogous means and post-process experimental data to obtain revised values of $\Delta h^{\circ}$ and $\Delta s^{\circ}$. Ultimately, the combination of $\Delta h^{\circ}, \Delta s^{\circ}$, and $F_{\text {int }}$ is used to predict the water uptake of the BZY bulk as a function of temperature and water partial pressure and the results are compared to various experimental findings.

\section{Methods}

\section{Equilibrium constants}

The non-ideal hydration equilibrium in reaction (2) can be separated into molar fractions and activity coefficients $\gamma_{i}$ and thus the non-ideal equilibrium constant $K$ is defined by eqn (5).

$$
K\left(T,\left[\mathrm{OH}_{\mathrm{O}}^{\bullet}\right]\right)=\frac{p^{\circ}}{p_{\mathrm{H}_{2} \mathrm{O}}} \cdot \frac{\left[\mathrm{OH}_{\mathrm{O}}^{\bullet}\right]^{2}}{\left[\mathrm{~V}_{\mathrm{O}}^{\bullet \bullet}\right]\left[\mathrm{O}_{\mathrm{O}}^{\times}\right]} \cdot \frac{\gamma_{\mathrm{OH}_{\mathrm{O}}}{ }^{2}}{\gamma_{\mathrm{v}_{\mathrm{O}}^{\bullet} \gamma_{\mathrm{O}_{\mathrm{O}}^{\times}}}}
$$

Alternatively, $K$ may be described by the ideal constant $K_{0}$ and a factor $K_{\text {int }}$ according to eqn (6). $K_{\text {int }}$ is the quotient of activity coefficients, which is here assumed to be defined exclusively by the ionic defect interactions.

$$
K\left(T,\left[\mathrm{OH}_{\mathrm{O}}^{\bullet}\right]\right)=K_{0}(T) \cdot K_{\mathrm{int}}\left(T,\left[\mathrm{OH}_{\mathrm{O}}^{\bullet}\right]\right)
$$

The site balance of the oxygen ion sublattice and the electroneutrality condition can be written as eqn (7) and (8), respectively.

$$
\begin{gathered}
{\left[\mathrm{V}_{\mathrm{O}}^{\bullet \bullet}\right]+\left[\mathrm{O}_{\mathrm{O}}^{\times}\right]+\left[\mathrm{OH}_{\mathrm{O}}^{\bullet}\right]=3} \\
2\left[\mathrm{~V}_{\mathrm{O}}^{\bullet \bullet}\right]+\left[\mathrm{OH}_{\mathrm{O}}^{\bullet}\right]=\left[\mathrm{Y}_{\mathrm{Zr}}^{\prime}\right]
\end{gathered}
$$

Combining eqn (5)-(8) yields the relation between the water partial pressure $p_{\mathrm{H}_{2} \mathrm{O}}$ and $\left[\mathrm{OH}_{\mathrm{O}}^{\bullet}\right]$ for the hydration of the interacting bulk system in eqn (9). While the separation of $K_{\text {int }}$ and $K_{0}$ is not accessible from experiments, it can be obtained from DFT/MMC simulations.

$$
\frac{p_{\mathrm{H}_{2} \mathrm{O}}}{p^{\circ}}=\frac{1}{K_{0} K_{\mathrm{int}}\left(\left[\mathrm{Y}_{\mathrm{Zr}}^{\prime}\right]-\left[\mathrm{OH}_{\mathrm{O}}^{\bullet}\right]\right)\left(6-\left[\mathrm{YH}_{\mathrm{Zr}}^{\prime}\right]-\left[\mathrm{OH}_{\mathrm{O}}^{\bullet}\right]\right)}
$$

The presented defect chemistry neglects other defects known to exist in the BZY system, namely, electronic hole defects under oxidizing conditions ${ }^{21-23}$ and yttrium dopants on the A sites of the perovskite. ${ }^{24,25}$ Additionally, a recent study ${ }^{26}$ suggests that BZY separates into an yttrium-rich and an yttrium-depleted phase around $10 \mathrm{~mol} \%$ of yttrium doping when cooled below $1600{ }^{\circ} \mathrm{C}$, which is also not considered in the present work.

\section{Calculating the free energy of interaction}

Following eqn (6), the apparent free enthalpy of hydration $\Delta G_{\text {app }}$ can be split into the ideal, non-interacting part $\Delta G^{\circ}$ and a defect interaction contribution $\Delta G_{\text {int }}^{\prime}$ per created $\mathrm{OH}_{\mathrm{O}}^{\bullet}$ defect as shown in eqn (10).

$$
\Delta G_{\text {app }}=\Delta G^{\circ}+2 \cdot \Delta G_{\text {int }}^{\prime}
$$

Accordingly, eqn (6) can then be expressed in generalized form of eqn (4) as shown in eqn (11).

$$
K\left(T,\left[\mathrm{OH}_{\mathrm{O}}^{\bullet}\right]\right)=\exp \left(\frac{-\Delta G^{\circ}}{k_{\mathrm{B}} T}\right) \exp \left(\frac{-2 \cdot \Delta G_{\mathrm{int}}^{\prime}}{k_{\mathrm{B}} T}\right)
$$

Neglecting volume changes, the approximation $\Delta G \approx \Delta F$ becomes applicable. Consequently, $K_{\text {int }}$ can be expressed by eqn (12) as a function of $\Delta F_{\text {int }}^{\prime}$.

$$
K_{\text {int }}\left(T,\left[\mathrm{OH}_{\mathrm{O}}^{\bullet}\right]\right)=\exp \left(\frac{-2 \cdot \Delta F_{\mathrm{int}}^{\prime}\left(T,\left[\mathrm{OH}_{\mathrm{O}}^{\bullet}\right]\right)}{k_{\mathrm{B}} T}\right)
$$

here, $\Delta F_{\text {int }}^{\prime}$ is the derivative of $\Delta F_{\text {int }}$ with respect to the proton count $N_{\mathrm{H}}$ and $F_{\text {int }}$ is the interaction contribution to the free 
energy $F$. Dry BZY with $F_{\text {int }}\left(N_{\mathrm{H}}=0\right)$ serves as the point of reference for $\Delta F_{\text {int }}$ according to eqn (13). A full derivation is available in the supplement of this article.

$$
\Delta F_{\text {int }}^{\prime}=\frac{\partial \Delta F_{\text {int }}}{\partial N_{\mathrm{H}}}=\frac{\partial\left(F_{\text {int }}\left(N_{\mathrm{H}}\right)-F_{\text {int }}\left(N_{\mathrm{H}}=0\right)\right)}{\partial N_{\mathrm{H}}}
$$

The system's free energy $F$ can be calculated using eqn (14) if the canonical partition function $Z$, eqn (15), is known.

$$
\begin{gathered}
F=-k_{\mathrm{B}} T \ln (Z) \\
Z=\int \Omega(E) \cdot \exp \left(\frac{-E}{k_{\mathrm{B}} T}\right) \mathrm{d} E
\end{gathered}
$$

here, $\Omega(E)$ is the total number of states with the energy $E$. With the MMC algorithm, samples of state distributions $M(E, T)$ fulfilling the relation in eqn (16) are obtained. However, the unknown proportionality constant $m$ prevents direct evaluation of $Z$ and consequently the calculation of $F$.

$$
m \cdot M(E, T)=\Omega(E) \cdot \exp \left(\frac{-E}{k_{\mathrm{B}} T}\right)
$$

A solution was suggested by Valleau and $\operatorname{Card}^{19}$ by connecting the MMC distribution $M(E, T)$ with a uniform random sample of the configurational space $\Omega^{\prime}(E)$ by a series of bridging distributions with small temperature deltas. The overlap of consecutive pairs of $M_{j}\left(E, T_{j}\right)$ and $M_{i}\left(E, T_{i}\right)$ yields $m_{i j}^{\prime}$ according to eqn (17), where $T_{j}>T_{i}$, and $M(E, T)$ converges to the random sample $\Omega^{\prime}(E)$ for $T \rightarrow \infty$.

$$
m_{i j}^{\prime}=\frac{\int M_{j}(E) \mathrm{d} E}{\int M_{i}(E) \cdot \exp \left(\frac{E}{k_{\mathrm{B}} T_{i}}-\frac{E}{k_{\mathrm{B}} T_{j}}\right) \mathrm{d} E}
$$

$F_{\text {int }}$ is then obtained from evaluating eqn (18) which allows evaluation of $\Delta F_{\text {int }}^{\prime}$ by eqn (13).

$$
F_{\text {int }}=-k_{\mathrm{B}} T \cdot \sum \ln \left(m_{i j}^{\prime}\right)
$$

A more detailed derivation of the principle is available in the supplement of this article and further explanation of the underlying mathematics can be found in the literature. ${ }^{19,20}$

\section{Computational setup}

In the MMC simulations, BZY is modelled using space group $P m \overline{3} m$ with a lattice constant of $4.23 \AA$ where the undoped

\begin{tabular}{|c|c|c|c|c|c|c|}
\hline \multirow{2}{*}{$\frac{\text { Site }}{\mathrm{Zr}}$} & \multicolumn{3}{|c|}{ Position (A, B, C) } & \multirow{2}{*}{$\frac{N}{1}$} & \multirow{2}{*}{$\frac{\text { Species }}{\mathrm{Zr}_{\mathrm{Zr}}^{\times}, \mathrm{Y}_{\mathrm{Zr}}^{\prime}}$} & \multirow{2}{*}{$\begin{array}{l}\text { Distribution } \\
\text { Random }\end{array}$} \\
\hline & $1 / 2$ & $1 / 2$ & $1 / 2$ & & & \\
\hline $\mathrm{O}$ & $1 / 2$ & $1 / 2$ & 0 & 3 & $\mathrm{O}_{\mathrm{O}}^{\times}, \mathrm{V}_{\mathrm{O}}^{\bullet \bullet}$ & MMC \\
\hline $\mathrm{H}_{\mathrm{i}}$ & 0.7636 & $1 / 2$ & 0 & 12 & $\mathrm{~V}_{\mathrm{i}}^{\times}, \mathrm{H}_{\mathrm{i}}^{\mathrm{O}}$ & MMC \\
\hline
\end{tabular}
barium site is omitted. According to DFT calculations, there are four possible proton positions at approximately $1 \AA$ distance around each oxygen site. ${ }^{16}$ Due to symmetry, the unit cell is thus fully described by the data shown in Table 1 .

Table 1 The unit cell data for BZY excluding the barium site
Here, the species $\mathrm{V}_{i}^{\times}$describes the absence of an interstitial proton $\mathrm{H}_{\mathrm{i}}^{\bullet}$. During simulation, only the $\mathrm{O}$ and $\mathrm{H}_{\mathrm{I}}$ sublattices are active, that is, $\mathrm{O}_{\mathrm{O}}^{\times} / \mathrm{V}_{\mathrm{O}}^{\bullet \bullet}$ and $\mathrm{V}_{\mathrm{i}}^{\times} / \mathrm{H}_{\mathrm{i}}^{\bullet}$ swaps are performed. $\mathrm{Zr}$ and $\mathrm{Y}$ are immobile and uniformly distributed at random at the Zr-sites prior to the simulation. This assumes that high temperature sintering leads to a uniform random distribution of the $\mathrm{Y}_{\mathrm{Zr}}^{\prime}$ defects in BZY and neglecting any $\mathrm{Y}_{\mathrm{Zr}}^{\prime}-\mathrm{Y}_{\mathrm{Zr}}^{\prime}$ interactions. However, it should be noted that recent research ${ }^{27}$ suggests that the repulsive $\mathrm{Y}_{\mathrm{Zr}}^{\prime}-\mathrm{Y}_{\mathrm{Zr}}^{\prime}$ interaction causes cation defect ordering even if very high sintering temperatures are used during sample preparation.

The energy landscape is modelled using pair interactions obtained from DFT calculations with the software VASP. ${ }^{28,29}$ The calculation method is described in previous publications. ${ }^{16,30}$ The energy change $\Delta E\left(\mathrm{~S}_{0} \rightarrow \mathrm{S}_{1}\right)$ between the initial state $\mathrm{S}_{0}$ and final state $S_{1}$ of an MMC exchange attempt is calculated as the summation of all pair energy contributions $E_{i j}(i \neq j)$ for the particles $i$ and $j$ of the two states as shown in eqn (19).

$$
\Delta E\left(S_{0} \rightarrow S_{1}\right)=\frac{1}{2} \cdot\left(\sum_{i} \sum_{j} E_{i j}\left(\mathrm{~S}_{1}\right)-\sum_{i} \sum_{j} E_{i j}\left(\mathrm{~S}_{0}\right)\right)
$$

The average internal energy of the system due to defect interaction $U_{\text {int }}$ is then obtained from a Monte Carlo step (MCS) average of the pair interaction summation in thermal equilibrium according to eqn (20), which is equivalent to the mean value of the recorded energy distribution $M(E, T)$.

$$
U_{\mathrm{int}}=\frac{1}{2} \cdot\left\langle\sum_{i} \sum_{j} E_{i j}\right\rangle_{\mathrm{MCS}}=M(E, T)_{E}
$$

All MMC simulations were performed with our in-house Monte Carlo software MOCASSIN ${ }^{31}$ using the built-in MMC multistage sampling routine. The solver simulates an annealing process for supercells by executing sequential runs at decreasing temperature $T$, from infinity $T_{\infty}$ to a lower limit $T_{\min }$. Two runs are performed at each sampled temperature: (i) an equilibration to ensure the system has reached thermal equilibrium; and (ii) a main run where the energy of the system $E_{\text {int }}$ is recorded to collect the $M(E, T)$ distributions. A factor $\alpha(T)$ manipulating the Boltzmann probability $P_{i}$ of a transition $i$ as shown in eqn (21) and (22) was introduced to model the temperature changes with gradually increasing sampling precision for lower values of $T$ during simulation.

$$
\begin{gathered}
\alpha(T)=\frac{T_{\text {min }}}{T} \\
P_{i}(T)=\exp \left(\frac{-\Delta E_{i} \cdot \alpha(T)}{k_{\mathrm{B}} T_{\min }}\right)
\end{gathered}
$$

Simulations were performed for a long and a shortrange interaction model in $20 \times 10 \times 10$ supercells of (Ba) $\mathrm{Zr}_{1-x} \mathrm{Y}_{x} \mathrm{O}_{3-0.5 x}$ at $x \in[0.1 ; 0.2 ; 0.3]$ using $T_{\min }=273 \mathrm{~K}$. The samples are named BZY10, BZY20, and BZY30, respectively. The alpha range $\alpha \in[0 \ldots 1.0]$ was sampled with the step size $\Delta \alpha=$ 0.01 . For each yttrium fraction, 29 relative hydration levels $\beta \in$ [0...1.0] were simulated, where $\beta=0$ and $\beta=1$ correspond to dry or fully hydrated material, respectively. The interval $\beta \in[0 \ldots 0.1]$ 
was sampled with a stepping of $\Delta \beta=0.01$ and all higher hydration levels with $\Delta \beta=0.05$. Each parameter set was run 48 times with different random seeds and starting conditions and $50 \times 10^{6}$ cycle outcomes per histogram were recorded with a mapping resolution of $0.01 \mathrm{eV}$. In each equilibration phase, $5 \times 10^{6}$ cycles were performed prior to the data collection. 140592 MMC distributions $M\left(E, T, N_{\mathrm{H}}\right)$ for each of the six pairs of yttrium fraction and DFT model were collected, totalling 843552 recorded MMC distributions.

\section{Results and discussion}

\section{DFT calculations}

DFT calculations were performed to complete our previously reported interaction energies. ${ }^{16}$ Pair interaction models with short-range and long-range cut-off radii are distinguished for the MMC simulations, labelled (S) and (L) in all following figures, respectively. All pair interactions and model boundaries are illustrated in Fig. 1.

Five types of interactions are considered in the MMC simulations, namely, $\mathrm{H}_{\mathrm{i}}^{\bullet}-\mathrm{H}_{\mathrm{i}}^{\bullet}, \mathrm{H}_{\mathrm{i}}^{\bullet}-\mathrm{V}_{\mathrm{O}}^{\bullet}, \mathrm{H}_{\mathrm{i}}^{\bullet}-\mathrm{Y}_{\mathrm{Zr}}^{\prime}, \mathrm{V}_{\mathrm{O}}^{\bullet \bullet}-\mathrm{Y}_{\mathrm{Zr}}^{\prime}$, and $\mathrm{V}_{\mathrm{O}}^{\bullet \bullet}-\mathrm{V}_{\mathrm{O}}^{\bullet \bullet}$. In general, $\mathrm{BZO}$ is highly symmetric and thus few unique interactions per unit range exist. However, there are four stable proton interstitial sites located around each oxygen site that are relevant to the hydration process, yielding a total of 24 unique $\mathrm{H}_{\mathrm{i}}^{\bullet}-\mathrm{H}_{\mathrm{i}}^{\bullet}$ geometries within a $5 \AA$ radius that can be distinguished in DFT calculations.

The $\mathrm{V}_{\mathrm{O}}^{\bullet \bullet}-\mathrm{Y}_{\mathrm{Zr}}^{\prime}$ interactions are strictly negative and converge towards zero at increasing distance as expected due to Coulomb's law. The $\mathrm{H}_{\mathrm{i}}^{\bullet}-\mathrm{Y}_{\mathrm{Zr}}^{\prime}$ interaction is also negative, however the first nearest neighbour attraction $(1 \mathrm{NN})(-0.3 \mathrm{eV})$ is slightly weaker than the $2 \mathrm{NN}$ interaction $(-0.37 \mathrm{eV})$. Also, in accordance with Coulomb's law, the interactions for $\mathrm{H}_{\mathrm{i}}^{\bullet}-\mathrm{H}_{\mathrm{i}}^{\bullet}, \mathrm{H}_{\mathrm{i}}^{\bullet}-\mathrm{V}_{\mathrm{O}}^{\bullet \bullet}$, and $\mathrm{V}_{\mathrm{O}}^{\bullet \bullet}-\mathrm{V}_{\mathrm{O}}^{\bullet \bullet}$ are mostly positive with a few exceptions that may be the result of structural relaxation of the supercells during DFT calculations. In the defective cell, the oxygen coordination octahedra around $\mathrm{Zr}$ sites can tilt, leading to a significant reduction of the energy of the supercell. If the energy reduction caused by the tilt overcompensates the Coulomb repulsion, then the $\mathrm{H}_{\mathrm{i}}^{\bullet}-\mathrm{H}_{\mathrm{i}}^{\bullet}, \mathrm{H}_{\mathrm{i}}^{\bullet}-\mathrm{V}_{\mathrm{O}}^{\bullet \bullet}$, and $\mathrm{V}_{\mathrm{O}}^{\bullet \bullet}-\mathrm{V}_{\mathrm{O}}^{\bullet \bullet}$ interactions may become slightly negative at certain positions.

There are interactions that show significant repulsive energies: (i) the strong $\mathrm{H}_{\mathrm{i}}^{\bullet}-\mathrm{V}_{\mathrm{O}}^{\bullet \bullet}$ repulsion at $1 \mathrm{NN}$ position $(2.57 \mathrm{eV})$, which means that creating isolated $\mathrm{H}_{\mathrm{i}}^{\bullet}$ defects rather than $\mathrm{OH}_{\mathrm{O}}^{\bullet}$ is extremely unfavourable; (ii) the $\mathrm{H}_{\mathrm{i}}^{\bullet}-\mathrm{H}_{\mathrm{i}}^{\bullet}$ repulsions at $1 \mathrm{NN}(1.21$ $\mathrm{eV}$ ) and $3 \mathrm{NN}(2.34 \mathrm{eV})$, which correspond to the unfavourable option of two $\mathrm{H}_{\mathrm{i}}^{\bullet}$ attaching to the same $\mathrm{O}_{\mathrm{O}}^{\times}$- formally creating an $\left(\mathrm{OH}_{2}\right)_{\mathrm{O}}^{\times}$water-like defect; and (iii) the strong $\mathrm{V}_{\mathrm{O}}^{\bullet \bullet}-\mathrm{V}_{\mathrm{O}}^{\bullet \bullet}$ repulsion at $2 \mathrm{NN}-\mathrm{b}(2.30 \mathrm{eV})$, corresponding to an interaction where a cation is located at the midpoint between the two $\mathrm{V}_{\mathrm{O}}^{\bullet \bullet}$ defects, which is analogously expected to greatly reduce the probability of two $\mathrm{V}_{\mathrm{O}}^{\bullet \bullet}$ forming this local configuration unless the concentration of $\mathrm{V}_{\mathrm{O}}^{\bullet \bullet}$ is very high.

The complete set of interaction tables with symmetryreduced reference geometries can be found in the supplement of this article.

\section{Internal and free energy of interactions}

The presented data is limited to the temperature set in the practically interesting range of $0{ }^{\circ} \mathrm{C}$ to $1000{ }^{\circ} \mathrm{C}$. The reference states for values of $\Delta U_{\text {int }}, \Delta F_{\text {int }}, \Delta S_{\text {int }}$ are the dry supercells with $\left[\mathrm{OH}_{\mathrm{O}}^{\bullet}\right]=0$ at the same temperature and yttrium content. Thus, these quantities describe the changes in defect interactions due to hydration.

Fig. 2 and 3 show the changes in internal energy $\Delta U_{\text {int }}$ and free energy $\Delta F_{\text {int }}$ for the BZY10 supercells using the short-range model, respectively. Both quantities are strictly negative, that is, interactions favour the hydration process, and the absolute values of $\Delta F_{\text {int }}$ are always less than $\Delta U_{\text {int }}$ as the defect interactions reduce the configurational space and thus the system loses configurational entropy. Within the applied DFT model, the energy decrease can be explained by the creation of
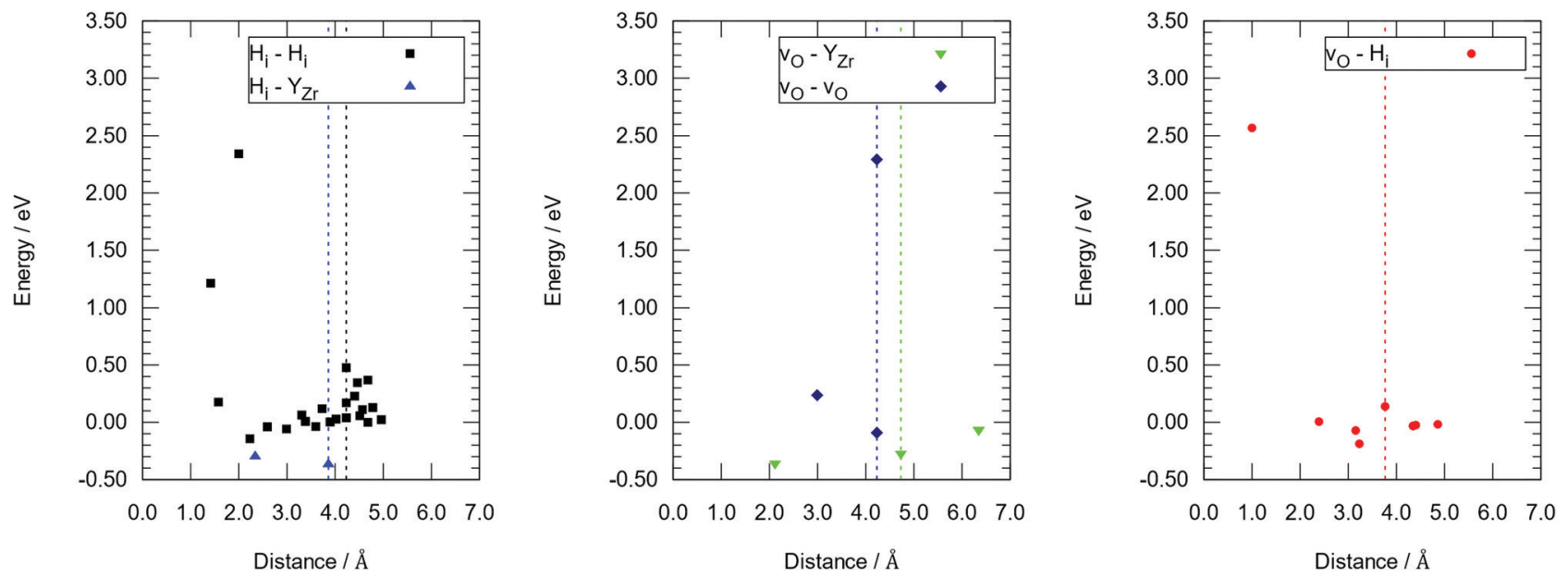

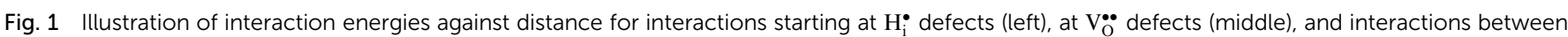
these two defects (right). Dashed lines indicate the maximum distances included in the short-range (S) model for each type of the interactions. 
$\beta$

$\begin{array}{lllllllllll}0 & 0.1 & 0.2 & 0.3 & 0.4 & 0.5 & 0.6 & 0.7 & 0.8 & 0.9 & 1\end{array}$

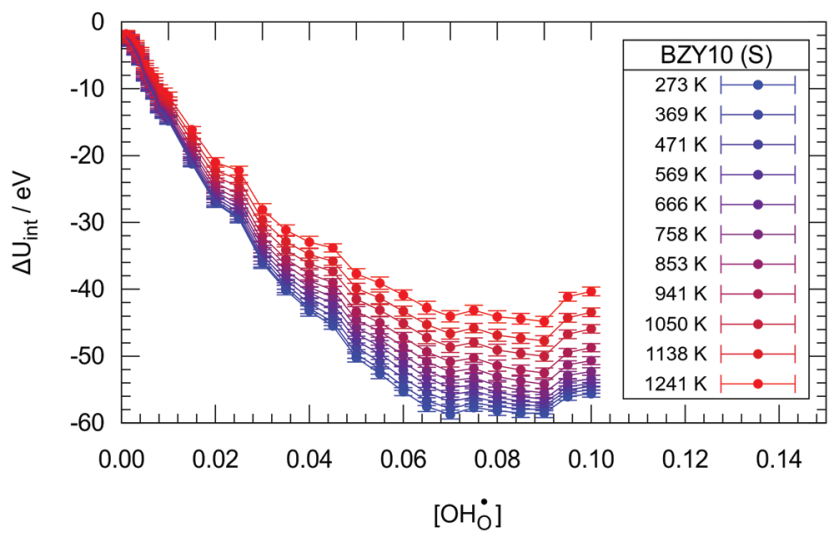

Fig. 2 The change in internal energy $\Delta U_{\text {int }}$ due to hydration in the BZY10 supercells using the short-range model. Lines are guide to the eye only.

$\beta$

$\begin{array}{lllllllllll}0 & 0.1 & 0.2 & 0.3 & 0.4 & 0.5 & 0.6 & 0.7 & 0.8 & 0.9 & 1\end{array}$

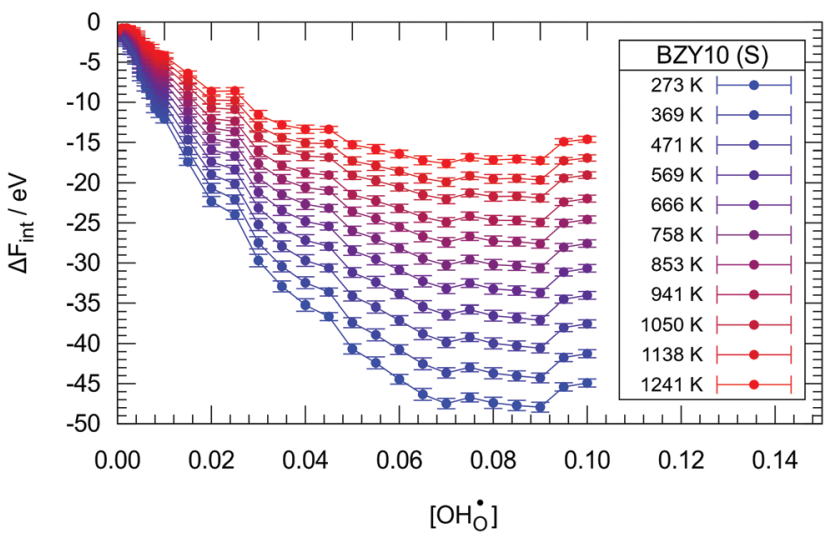

Fig. 3 The change in free energy $\Delta F_{\text {int }}$ due to hydration in the BZY10 supercells using the short-range model. Lines are guide to the eye only.

attractive $\mathrm{H}_{\mathrm{i}}^{\bullet}-\mathrm{Y}_{\mathrm{Zr}}^{\prime}$ interactions $(-0.30 \mathrm{eV}$ at $1 \mathrm{NN}$ and $-0.37 \mathrm{eV}$ at $2 \mathrm{NN}$ ) that overcompensate the energy increase resulting from the removal of attractive $\mathrm{V}_{\mathrm{O}}^{\bullet-}-\mathrm{Y}_{\mathrm{Zr}}^{\prime}$ interactions $(-0.36 \mathrm{eV}$ at $1 \mathrm{NN}$, $-0.28 \mathrm{eV}$ at $2 \mathrm{NN},-0.07 \mathrm{eV}$ at $3 \mathrm{NN}$ ). This is further supported by the removal of $\mathrm{V}_{\mathrm{O}}^{\bullet \bullet}$ defects reducing the number of repelling $\mathrm{V}_{\mathrm{O}}^{\bullet \bullet}-\mathrm{V}_{\mathrm{O}}^{\bullet \bullet}$ interactions $(0.24 \mathrm{eV}$ at $1 \mathrm{NN}$, up to $2.30 \mathrm{eV}$ at $2 \mathrm{NN})$ that occur when multiple $\mathrm{V}_{\mathrm{O}}^{\bullet \bullet}$ defects aggregate at the same $\mathrm{Y}_{\mathrm{Zr}}^{\prime}$ defect.

The contribution per defect decreases with increasing hydration level and $\Delta U_{\text {int }}$ and $\Delta F_{\text {int }}$ both traverse minima around $\left[\mathrm{OH}_{\mathrm{O}}^{\bullet}\right]=0.08$ independent of temperature. This allows two assumptions: (i) gradual hydration progressively becomes less favourable; and (ii) for hydration above $\left[\mathrm{OH}_{\mathrm{O}}^{\bullet}\right]=0.08$, the interactions of ionic defects have an impeding effect on further hydration.

The interaction-induced changes in configurational entropy $\Delta S_{\text {int }}$ are obtained using the relation $F=U-T S$. The results for BZY10 with the short-range DFT model are illustrated in Fig. 4. Two expected features are visible: (i) all values are negative, as $\beta$

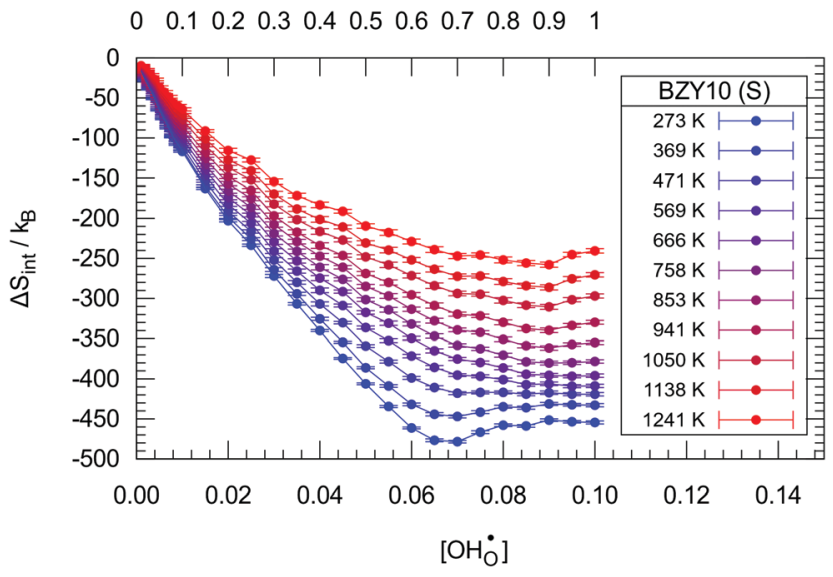

Fig. 4 The change in entropy $\Delta S_{\text {int }}$ due to hydration in the BZY10 supercells using the short-range model. Lines are guide to the eye only.

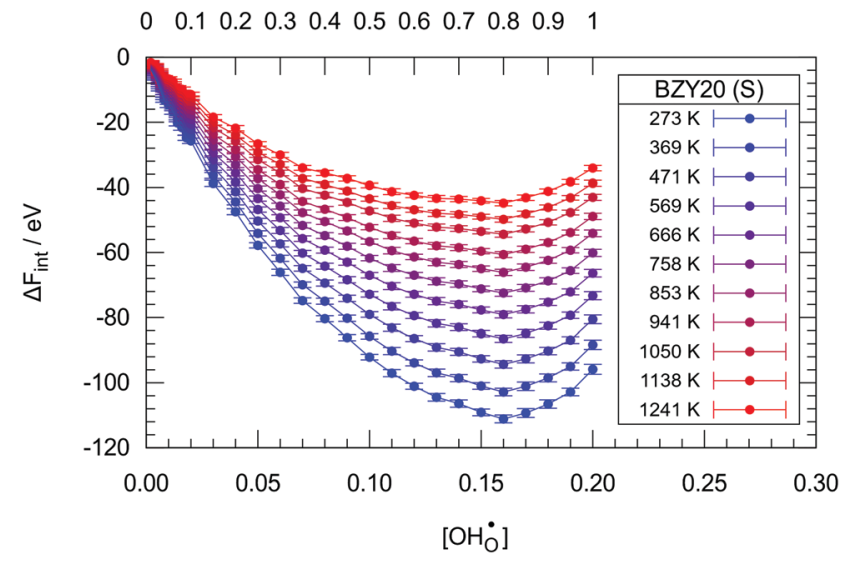

Fig. 5 The change in free energy $\Delta F_{\text {int }}$ due to hydration in the BZY20 supercells using the short-range model. Lines are guide to the eye only.

$\beta$

$\begin{array}{lllllllllll}0 & 0.1 & 0.2 & 0.3 & 0.4 & 0.5 & 0.6 & 0.7 & 0.8 & 0.9 & 1\end{array}$

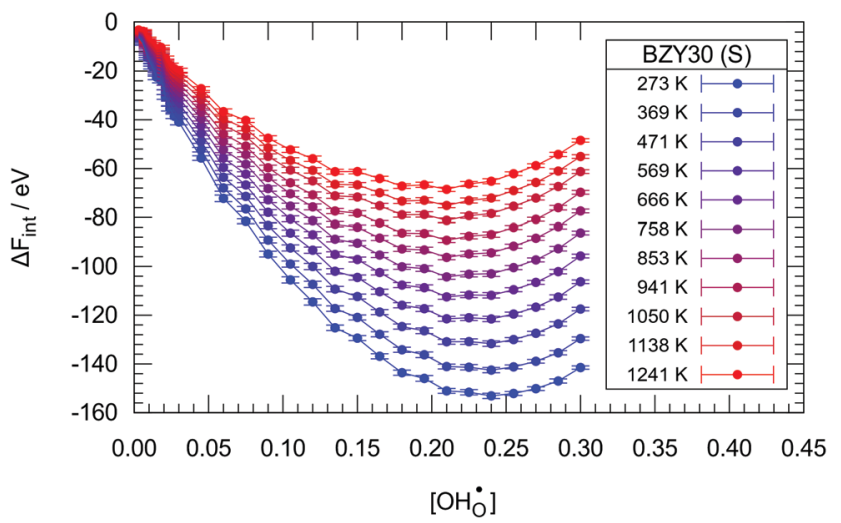

Fig. 6 The change in internal energy $\Delta F_{\text {int }}$ due to hydration in the BZY30 supercells using the short-range model. Lines are guide to the eye only. 
interactions cause ordering of the system by restricting the accessibility of configurations; and (ii) the loss of entropy is less pronounced for increasing temperature, as more thermal fluctuation reduces the order of defects. For low temperatures, $\Delta S_{\text {int }}$ traverses an unexpected minimum which could be related to low-temperature cluster formation. This is however hard to verify due to the small effect.

The analogously obtained results for $\Delta F_{\text {int }}$ in the BZY20 and BZY30 supercells using the short-range model are illustrated in Fig. 5 and 6, respectively. The minima for BZY20 are independent of temperature, lie very close to $\left[\mathrm{OH}_{\mathrm{O}}^{\circ}\right]=0.16$, and thus behave analogously to the BZY10 simulations. In contrast, the BZY30 simulations show a slightly temperature dependent behaviour where the energy minimum is shifted towards lower hydration levels with rising temperature. Thus, the hindering of gradual hydration starts at lower relative hydration levels in contrast to BZY10 and BZY20. The results for the long-range model are similar and can be found in the supplement. In general, the long-range interaction data indicate a less favouring effect on hydration than the short-range model.

\section{Calculation of the $\boldsymbol{K}_{\text {int }}$ datasets}

The $\Delta F_{\text {int }}^{\prime}$ datasets are obtained by evaluating eqn (13) using a third order polynomial fitted to the simulated $\Delta F_{\text {int }}$ data. The fitting is only performed to obtain smooth functions for $\Delta F_{\text {int }}$ and to reduce the data fluctuations in the derivative $\partial \Delta F_{\text {int }} \partial N_{\mathrm{H}}$. The results for $\Delta F_{\text {int }}^{\prime}$ using the short-range model are summarized in Fig. 7 and the results for the long-range model can be found in the supplement.

Fig. 7 reveals two expected properties: (i) the magnitude of $\Delta F_{\text {int }}^{\prime}$ declines with increasing temperature and proton concentration, as the influence of the $T \Delta S_{\text {int }}$ term on $\Delta F_{\text {int }}$ increases and partially compensates for the negative enthalpic term; and (ii) the curves flatten with rising temperature due to the weakening of the influence of defect interactions.

Evaluation of eqn (12) with the $\Delta F_{\text {int }}^{\prime}$ data yields the $K_{\text {int }}$ datasets. The results for BZY10, BZY20, and BZY30 using the short-range model are summarized in Fig. 8. The differences

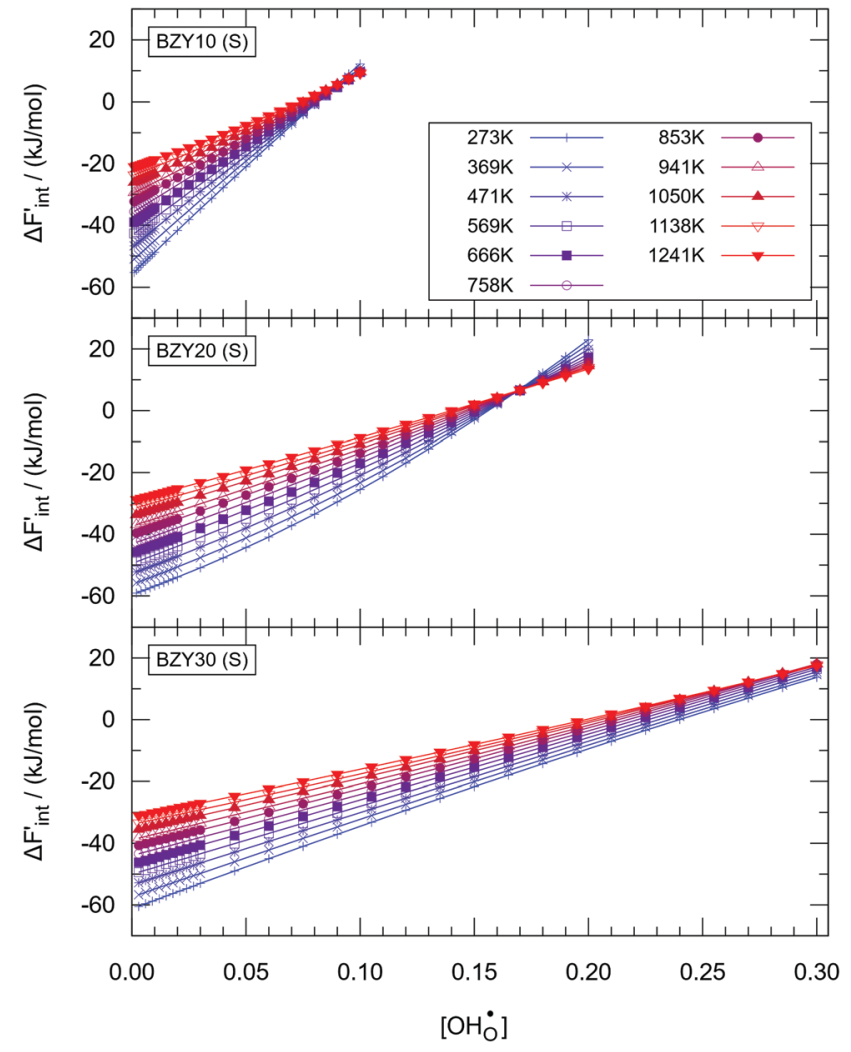

Fig. 7 The calculated values of $\Delta F_{\text {int }}^{\prime}$ for the short-range model in BZY10 (top), BZY20 (middle), and BZY30 (bottom). Lines are guide to the eye only.

between the yttrium fractions are much smaller than the divergences due to proton defects or temperature changes, which agrees with the experimental observation that $x_{\mathrm{Y}}$ affects the equilibrium constants to a minor degree only. ${ }^{3,5,32}$

The distinct zones of interest are $K_{\mathrm{int}}>1, K_{\mathrm{int}} \approx 1$, and $K_{\mathrm{int}}<1$, which imply that proton incorporation is above, equal, or below the ideal case without defect interactions, respectively. Thus, the shown trends in $K_{\text {int }}$ are expected when compared to the ideal model, that is: (i) regardless of temperature, it is generally more

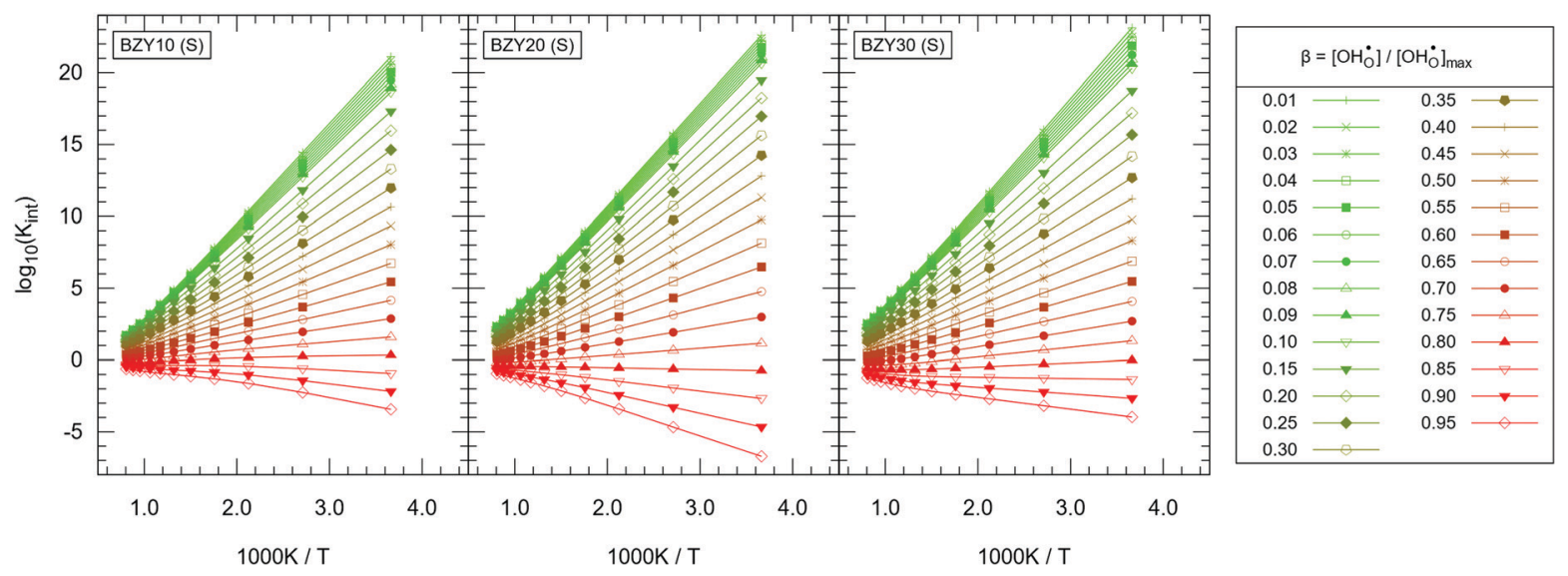

Fig. 8 The $K_{\text {int }}$ factors for BZY10 (left), BZY20 (middle), and BZY30 (right) as obtained using the short-range interaction model. The data is normalized to the maximum proton concentration $\left[\mathrm{OH}_{\mathrm{O}}^{\circ}\right]_{\max }=x_{\mathrm{Y}}$ of each simulation. Lines are guide to the eye only. 
favourable to have at least a few protons incorporated into the system (green curves); (ii) it is significantly harder to push the material towards very high levels of hydration (red curves); and (iii) the gap in $K_{\text {int }}$ between low and high $\left[\mathrm{OH}_{\mathrm{O}}^{\bullet}\right]$ is decreasing by orders of magnitude from $0{ }^{\circ} \mathrm{C}$ to $1000{ }^{\circ} \mathrm{C}$ due to the weakening of the influence of defect interactions with rising temperature. Since the spread in $K_{\text {int }}$ gradually declines with rising temperature approaching $K_{\mathrm{int}} \approx 1$, fitting of experimental results should be done for high temperatures. Optionally, $K_{\mathrm{int}} \approx 1$ is reached for very high proton concentrations around $0.80-0.90$ - depending on the yttrium fraction - over a wide temperature range.

\section{Extraction of $\Delta \boldsymbol{h}^{\circ}$ and $\Delta \boldsymbol{s}^{\circ}$ from experimental data}

The presented simulation data can be used to evaluate and postprocess experimental results. By rewriting eqn (6) into eqn (23), it is apparent that the $\left[\mathrm{OH}_{\mathrm{O}}^{\bullet}\right]$ dependency of experimental $K\left(T,\left[\mathrm{OH}_{\mathrm{O}}^{\bullet}\right]\right)$ values can be eliminated using the $K_{\text {int }}$ datasets from MMC. This yields the $\left[\mathrm{OH}_{\mathrm{O}}^{\bullet}\right]$ independent $K_{0}(T)$ data and consequently revised values of $\Delta h^{\circ}$ and $\Delta s^{\circ}$ can be obtained from the Arrhenius formalism. In return, the applicability of the separation principle is implicitly validated by this approach.

$$
K_{0}(T)=\frac{K\left(T,\left[\mathrm{OH}_{\mathrm{O}}^{\bullet}\right]\right)}{K_{\mathrm{int}}\left(T,\left[\mathrm{OH}_{\mathrm{O}}^{\bullet}\right]\right)}
$$

A detailed experimental analysis for BZY10 was performed by Imai et $a l .{ }^{4}$ We use their data for comparison and analysis based on eqn (23) and consequently to obtain revised estimations for $\Delta h^{\circ}$ and $\Delta s^{\circ}$. Imai et al. investigated multiple hydration levels at each measured temperature. Using interpolated values of $K_{\mathrm{int}}$ matching their conditions, the generated $K_{0}(T)$ dataset should ideally be independent of $\left[\mathrm{OH}_{\mathrm{O}}^{\bullet}\right]$ at each temperature. The $K_{0}$ values extracted from the experimental data are summarized in Fig. 9.

None of the two models accomplishes a constant $K_{0}$ for each temperature but the $\left[\mathrm{OH}_{\mathrm{O}}^{\bullet}\right]$ dependence is corrected in the right direction compared to $K$. $K_{0}$ no longer shows a strict decline with gradual hydration. Instead, for $873 \mathrm{~K}$ and $973 \mathrm{~K}$ the data shows slightly positive slopes and for $1073 \mathrm{~K}$ and $1173 \mathrm{~K}$ the curves are almost constant. Thus, the method seems to overestimate the correction for lower temperatures. The trends for low temperatures are not unexpected as pair interaction models should yield a progressively less accurate description for higher defect concentrations, which are present in BZY at low temperatures and/or at high water partial pressures.

Overall, considering that $K_{0}$ inherits the experimental fluctuations from $K$, the results indicate that the $\left[\mathrm{OH}_{\mathrm{O}}^{\bullet}\right]$ dependence is greatly reduced and that the ionic defect interactions are the primary reason for non-ideal behaviour observed in the experimental data. Fig. 10 shows the Arrhenius diagrams obtained from the temperature averages of $K_{0}$. While the systematic differences in the $K$ data by Imai et al. prevent a direct evaluation, the evaluation of calculated $K_{0}(T)$ data shows an Arrhenius behaviour within the margin of error.

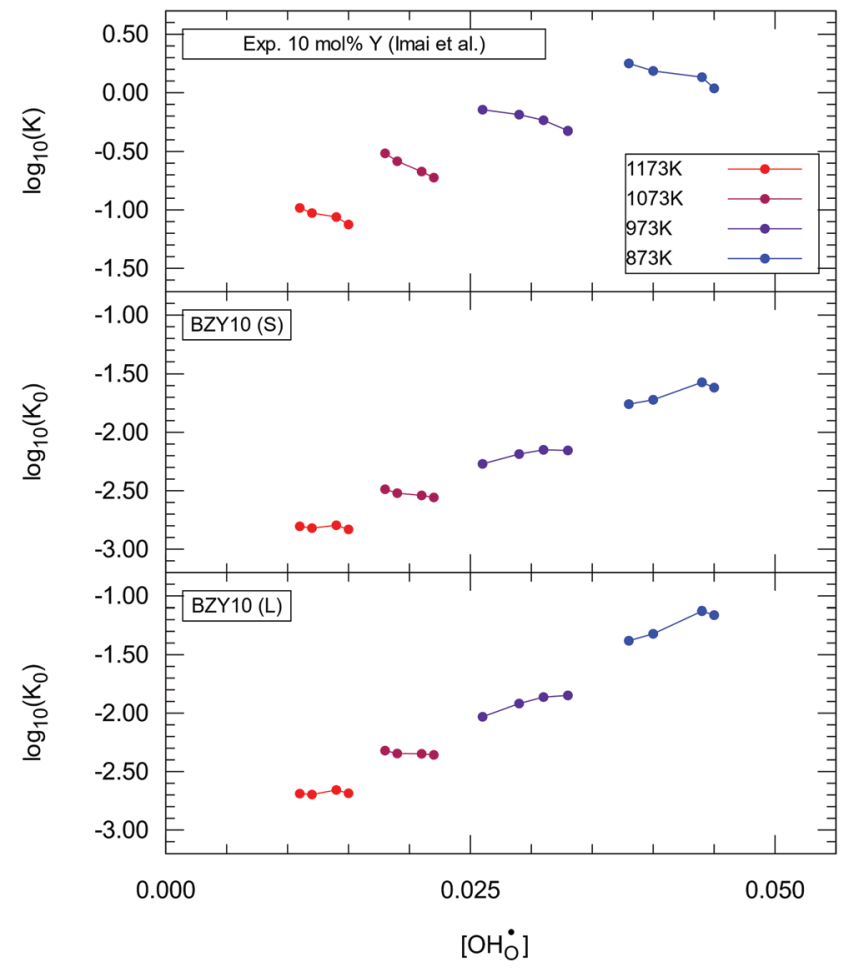

Fig. 9 The experimental equilibrium constants $K$ by Imai et al. (top) in comparison to the $K_{0}$ values yielded by division with $K_{\text {int }}$ data from the short-range (middle) and the long-range model data (bottom). Lines are guide to the eye only.

Fitting yields $\Delta h^{\circ}=-75 \pm 4 \mathrm{~kJ} \mathrm{~mol}^{-1}$ and $\Delta s^{\circ}=-118 \pm$ $4 \mathrm{~J} \mathrm{~mol}^{-1} \mathrm{~K}^{-1}$ for the short-range model, and $\Delta h^{\circ}=-94 \pm$ $5 \mathrm{~kJ} \mathrm{~mol}{ }^{-1}$ and $\Delta s^{\circ}=-132 \pm 5 \mathrm{~J} \mathrm{~mol}^{-1} \mathrm{~K}^{-1}$ for the longrange model. The obtained values of $\Delta h^{\circ}$ and $\Delta s^{\circ}$ lie within the data range as reported in the literature ${ }^{2,5,6}$ for experiments conducted above $500{ }^{\circ} \mathrm{C}$. This is expected, as there are few interacting protons present and $K_{\text {int }}$ has a minor effect at high temperatures.

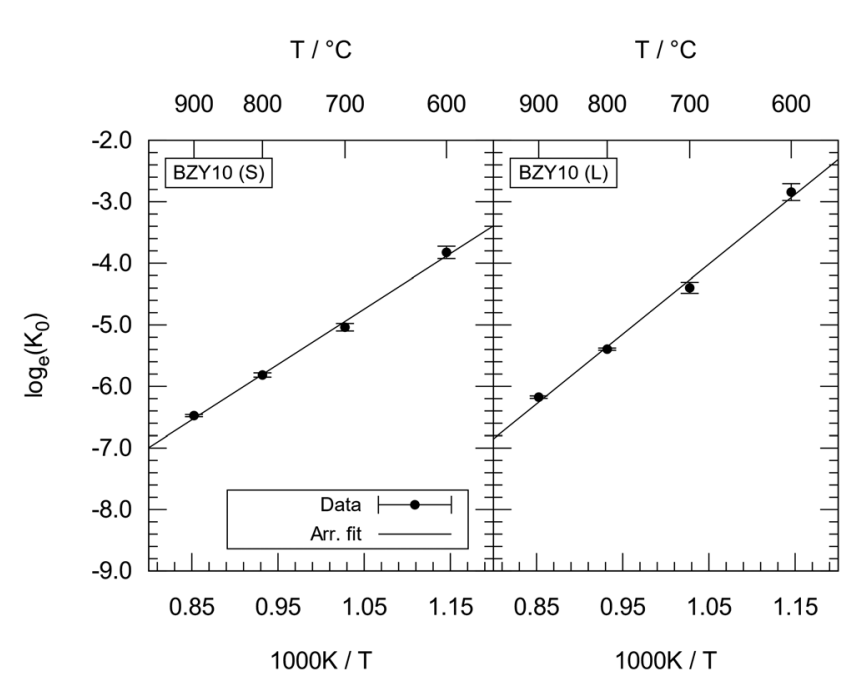

Fig. 10 Arrhenius diagrams for temperature averages of $K_{0}$ as obtained from the short-range model (left) and long-range model (right). 


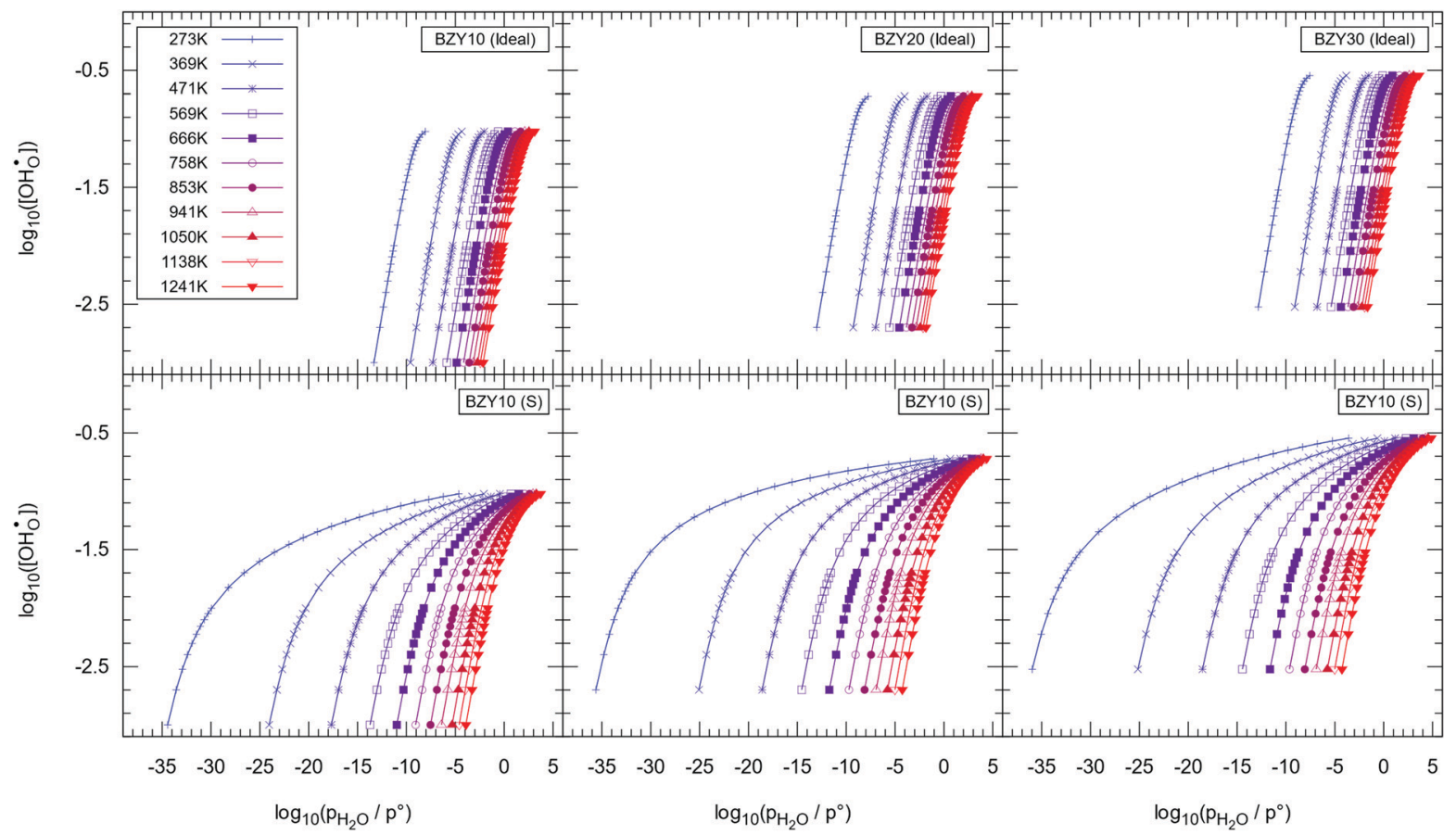

Fig. 11 Illustration of $\left[\mathrm{OH}_{\mathrm{O}}^{\circ}\right]$ against $p_{\mathrm{H}_{2} \mathrm{O}}$ for BZY10 (left), BZY2O (middle), and BZY30 (right) using idealized data calculated with $\Delta s^{\circ}, \Delta h^{\circ}$ (top) compared to the non-ideal curves after correction of the ideal data with the $K_{\text {int }}$ datasets (bottom). Lines are guide to the eye only.

\section{Dependence of $\left[\mathrm{OH}_{\mathrm{O}}^{\bullet}\right]$ on water partial pressure}

The simulated $K_{\text {int }}$ in combination with extracted $K_{0}$ allow prediction of the $p_{\mathrm{H}_{2} \mathrm{O}}$ vs. $\left[\mathrm{OH}_{\mathrm{O}}^{*}\right]$ relations (hydration isotherms) for the full temperature and $p_{\mathrm{H}_{2} \mathrm{O}}$ range. Fig. 11 shows $\left[\mathrm{OH}_{\mathrm{O}}^{\circ}\right]$ against $p_{\mathrm{H}_{2} \mathrm{O}}$ for the short-range model calculated with eqn (9) in comparison to the curves calculated from $K_{0}$. Based on the experimental observation that $x_{\mathrm{Y}}$ has a minor effect on the equilibrium constants, ${ }^{32} \Delta h^{\circ}$ and $\Delta s^{\circ}$ are also adopted for
BZY20 and BZY30. The illustration for the long-range model can be found in the supplement of the article.

Fig. 11 reveals the strong deviation from an ideal behaviour with the largest divergence at $0{ }^{\circ} \mathrm{C}$ to $200{ }^{\circ} \mathrm{C}$, which is expected as the hydration of BZY is exothermic and defect interactions have the largest impact at low temperatures. The data suggests almost full hydration at $p_{\mathrm{H}_{2} \mathrm{O}}<0.1 \mathrm{~Pa}$ and $T=0{ }^{\circ} \mathrm{C}$. However, slow kinetics with long equilibration times could be expected under these conditions and thus the data illustrates the limiting
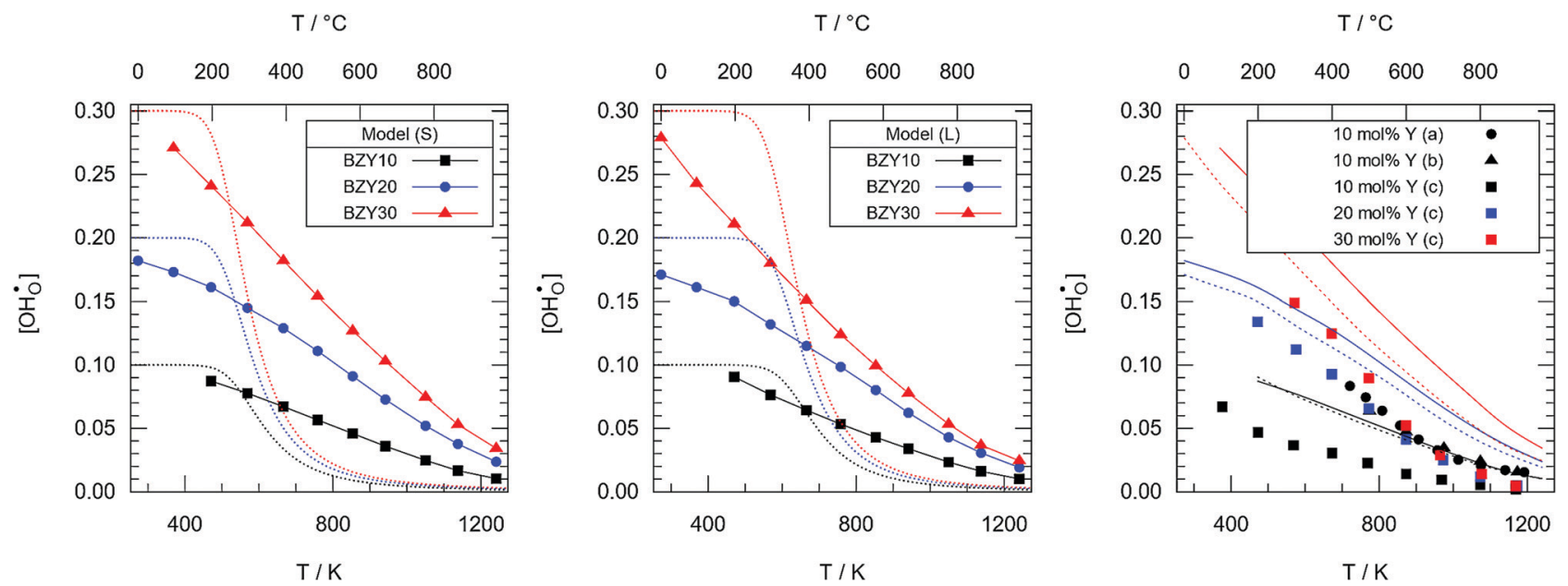

Fig. 12 Illustration of $\left[\mathrm{OH}_{\mathrm{O}}^{*}\right]$ against $T$ (hydration isobars) for BZY10, BZY20, and BZY30 at 23 mbar water partial pressure using the $K_{\text {int }}$ post-processed data from the short-range model (left) and the long-range model (middle) compared to measured experimental results (right) by Kreuer et al. (a) [10 mol\% Y at $23 \mathrm{mbar}$ ], Imai et al. (b) [10 mol\% Y at $23 \mathrm{mbar}$, and Gonçalves et al. (c) [10, 20, 30 mol\% Y at $19 \mathrm{mbar}$. The dotted lines (left, middle) indicate the ideal equilibrium models as calculated from the obtained $\Delta h^{\circ}$ and $\Delta s^{\circ}$ without the $K_{\text {int }}$ correction. The solid and dashed lines (right) indicate the corrected $S$ and $\mathrm{L}$ model, respectively. Other lines are guide to the eye only. 
case, which might not be accessible in experiments. Additionally - as mentioned previously - the method seems to progressively overestimate the $K_{\text {int }}$ data with decreasing temperatures.

For a typical experimental water partial pressure of $23 \mathrm{mbar}$, the hydration isobar $\left[\mathrm{OH}_{\mathrm{O}}^{\bullet}\right](T)$ according to eqn (9) is presented in Fig. 12 for both models in comparison to ideal curves according to eqn (4) using $\Delta s^{\circ}$ and $\Delta h^{\circ}$ as determined above. Various experimental results from literature which were obtained at comparable yttrium fraction and water partial pressure are shown for comparison. The following features can be identified: (i) the data including $K_{\text {int }}$ strongly deviates from the ideal curves improving or hindering the hydration at the high and low temperature regions, respectively; (ii) the favouring effect of the long-range model is smaller than for the short range model; and (iii) there is an excellent agreement of the BZY10 curves with the experimental data by Imai et $a l^{4}{ }^{4}$ and Kreuer et $a .^{3}$ - measured at $23 \mathrm{mbar}$ and $10 \mathrm{~mol} \% \mathrm{Y}$ content - and a good qualitative agreement of the BZY20 and BZY30 curves with the data by Gonçalves et al. ${ }^{5}$ - measured at $19 \mathrm{mbar}$ and $20 \mathrm{~mol} \%$ and $30 \mathrm{~mol} \%$ yttrium, respectively. However, the deviation of their results for $10 \mathrm{~mol} \%$ yttrium from the data by the other groups is unexpected considering the minor change in $p_{\mathrm{H}_{2} \mathrm{O}}$. The affiliated MMC data also suggests higher $\left[\mathrm{OH}_{\mathrm{O}}^{\bullet}\right]$ saturation than measured by Gonçalves et al. The differences for low temperatures might originate from the mentioned issue of slow kinetics leading to different degrees of hydration.

In general, the inclusion of defect interactions in the form of $K_{\text {int }}$ leads to greatly improved description of the experimental data compared to the ideal model.

\section{Conclusions}

The non-ideal behaviour of barium zirconate hydration is an often-discussed issue in the literature. In this contribution, large-scale multistage MMC simulations based on a DFT derived pair interaction model were applied to estimate the influence of defect interactions on the hydration of BZY. The applied separation into interacting and non-interacting contributions by calculating the free energy of interaction $\Delta F_{\text {int }}$ allowed us to obtain estimations for the quotient of the activity coefficients condensed in the factor $K_{\text {int }}$. The post-processing of experimental $K$ data to obtain revised values of $K_{0}, \Delta h^{\circ}$, and $\Delta s^{\circ}$ successfully yielded $\left[\mathrm{OH}_{\mathrm{O}}^{\bullet}\right](T)$ datasets providing improved quantitative agreement with experimental data compared to the ideal solution model for BZY10, BZY20, and BZY30. Increasing the interaction range slightly improves the agreement of the MMC simulations with experimental results for BZY20 and BZY30, however the benefit-cost ratio rapidly diminishes.

The applied method investigates interacting ensembles using fixed-position MMC simulations with DFT pair interaction models, while neglecting the influences of electron hole defects, yttrium defects on A sites, and surface effects. Structural relaxation of the system is implicitly considered during the DFT calculations of the pair interactions, while further lattice distortion of specific configurations is not considered in the MMC simulations. Considering the excellent agreement with experimental data, the present results indicate that the non-ideality of the bulk water uptake can be explained mostly by the ionic defect interactions affecting both low and high temperature regimes.

The often-published conclusion that low temperature data shows ideal behaviour is not directly confirmed. Instead, there are strong deviations from ideal behaviour observed for the entire temperature regime. However, the influence of $K_{\text {int }}$ on the equilibrium constant does not change over several orders of magnitude, that is, $K_{\text {int }}\left(T,\left[\mathrm{OH}_{\mathrm{O}}^{\bullet}\right]\right) \approx$ const., within the very high and very low temperature regimes. Thus, these regions can be acceptably fitted using an Arrhenius model as commonly done in experimental studies. The largest impact of interactions is found for low temperature and very low proton fraction or water partial pressure. However, this equilibrium might not be observable in experiments as the kinetics could be a limiting factor and $K_{\text {int }}$ seems to be progressively overestimated with decreasing temperature.

We conclude that the simulated data seems to predict the experimental trends very well and can be used to correct and analyse experimental data by including the ionic defect interactions into the BZY hydration model.

\section{Author contributions}

S. E. implemented the Monte Carlo system MOCASSIN ${ }^{31}$ with the MMC multistage sampling and performed all MMC simulations and data evaluation. F. M. D. performed the DFT calculations and supplied the pair interaction data. S. G. initiated and supervised the research. All authors have contributed to the preparation of the manuscript.

\section{Conflicts of interest}

There are no conflicts to declare.

\section{Acknowledgements}

The authors gratefully acknowledge the computing time granted by the JARA Vergabegremium and provided on the JARA Partition part of the supercomputer CLAIX at RWTH Aachen. We thank Prof. Manfred Martin from RWTH Aachen University for bringing our attention to the topic and for helpful discussions.

\section{References}

1 F. A. Kröger and H. J. Vink, in Solid state physics, ed. F. Seitz and D. Turnbull, Academic Press, New York, 1956, pp. 307435.

2 K. D. Kreuer, S. Adams, W. Münch, A. Fuchs, U. Klock and J. Maier, Solid State Ionics, 2001, 145, 295-306.

3 K. D. Kreuer, Annu. Rev. Mater. Res., 2003, 33, 333-359. 
4 G. Imai, T. Nakamura and K. Amezawa, Solid State Ionics, 2017, 303, 12-15.

5 M. D. Gonçalves, A. Mielewczyk-Gryń, P. S. Maram, Ł. Kryścio, M. Gazda and A. Navrotsky, J. Phys. Chem. C, 2020, 124, 11308-11316.

6 T. Schober, Solid State Ionics, 2000, 127, 351-360.

7 K. D. Kreuer, Solid State Ionics, 1999, 125, 285-302.

8 J. A. Dawson, J. A. Miller and I. Tanaka, Chem. Mater., 2015, 27, 901-908.

9 S. J. Stokes and M. S. Islam, J. Mater. Chem., 2010, 20, 6258.

10 Y. Yamazaki, F. Blanc, Y. Okuyama, L. Buannic, J. C. LucioVega, C. P. Grey and S. M. Haile, Nat. Mater., 2013, 12, 647-651.

11 K. Toyoura, T. Fujii, N. Hatada, D. Han and T. Uda, J. Phys. Chem. C, 2019, 123, 26823-26830.

12 N. Kochetova, I. Animitsa, D. Medvedev, A. Demin and P. Tsiakaras, RSC Adv., 2016, 6, 73222-73268.

13 K. Kreuer, Solid State Ionics, 2000, 136-137, 149-160.

14 P. Raiteri, J. D. Gale and G. Bussi, J. Phys.: Condens. Matter, 2011, 23, 334213.

15 M. Björketun, P. Sundell, G. Wahnström and D. Engberg, Solid State Ionics, 2005, 176, 3035-3040.

16 F. M. Draber, C. Ader, J. P. Arnold, S. Eisele, S. Grieshammer, S. Yamaguchi and M. Martin, Nat. Mater., 2020, 19, 338-346.

17 N. Metropolis and S. Ulam, J. Am. Stat. Assoc., 1949, 44, $335-341$.

18 N. Metropolis, A. W. Rosenbluth, M. N. Rosenbluth, A. H. Teller and E. Teller, J. Chem. Phys., 1953, 21, 1087-1092.
19 J. P. Valleau and D. N. Card, J. Chem. Phys., 1972, 57, 5457-5462.

20 S. Grieshammer and M. Martin, J. Mater. Chem. A, 2017, 5, 9241-9249.

21 K. Noromura and H. Kageyama, Solid State Ionics, 2007, 178, 661-665.

22 D. Han, Y. Noda, T. Onishi, N. Hatada, M. Majima and T. Uda, Int. J. Hydrogen Energy, 2016, 41, 14897-14908.

23 D. Han and T. Uda, J. Mater. Chem. A, 2018, 6, 18571-18582.

24 D. Han, K. Kishida, K. Shinoda, H. Inui and T. Uda, J. Mater. Chem. A, 2013, 1, 3027.

25 R. Sažinas, M. F. Sunding, A. Thøgersen, I. Sakaguchi, T. Norby, T. Grande and J. M. Polfus, J. Mater. Chem. A, 2019, 7, 3848-3856.

26 K. Ueno, N. Hatada, D. Han, K. Toyoura and T. Uda, J. Solid State Electrochem., 2020, 24, 1523-1538.

27 S. Kasamatsu, O. Sugino, T. Ogawa and A. Kuwabara, J. Mater. Chem. A, 2020, 8, 12674-12686.

28 G. Kresse and J. Furthmüller, Phys. Rev. B: Condens. Matter Mater. Phys., 1996, 54, 11169-11186.

29 G. Kresse and D. Joubert, Phys. Rev. B: Condens. Matter Mater. Phys., 1999, 59, 1758-1775.

30 S. Grieshammer, B. O. H. Grope, J. Koettgen and M. Martin, Phys. Chem. Chem. Phys., 2014, 16, 9974-9986.

31 S. Eisele and S. Grieshammer, J. Comput. Chem., 2020, 41, 2663-2677.

32 Y. Yamazaki, P. Babilo and S. M. Haile, Chem. Mater., 2008, 20, 6352-6357. 